\title{
Curriculum Pneumologicum 2005
}

Curriculum Pneumologicum 2005. Part 2

\section{Curriculum Pneumologicum (Fortsetzung aus Heft 1/2006)}

$\mathrm{W}=$ wichtig, 1 = muss man wissen, 2 = sollte man wissen, 3 = Spezialwissen

\begin{tabular}{|c|c|c|c|c|}
\hline Ziffer & Kapitel & Neuere Entwicklungen/Erkenntnisse & Literaturempfehlung & $w$ \\
\hline $\mathbf{E}$ & Umwelt/Arbeitswelt und Lunge & & & \\
\hline \multirow[t]{3}{*}{1} & Tabakrauchen (aktiv/passiv) & & & 1 \\
\hline & $\begin{array}{l}\text { Während die Risiken des Aktivrauchens } \\
\text { dem interessierten Pneumologen satt- } \\
\text { sam bekannt sind, haben sich die Er- } \\
\text { kenntnisse über die Schädlichkeit des } \\
\text { Passivrauchens in den letzten Jahren } \\
\text { stark erweitert. }\end{array}$ & $\begin{array}{l}\text { - Passivrauchen erhöht das Risiko für Lungenkrankheiten } \\
\text { allgemein (OR 1.30, 9\%), } \\
\text { - für Lungenkrebs im Erwachsenenalter, } \\
\text { - bei kindlicher Passivrauchexposition im Speziellen (OR } \\
\text { 3.63, 95\%) }\end{array}$ & $\begin{array}{l}\text { - Jöckel KH: } \\
\text { Gesundheitsrisiken durch Passivrauchen. } \\
\text { Deutsches Ärzteblatt 2000; 97: } 2417\end{array}$ & \\
\hline & $\begin{array}{l}\text { Die „Anti-Tabakfront“ ist jetzt auch in } \\
\text { Deutschland in Bewegung gekommen: } \\
\text { Erhöhung der Tabaksteuer, Arbeitstät- } \\
\text { tenverordnung zum Schutz gegen pas- } \\
\text { sives Raucher und zahlreiche Initiativen } \\
\text { von Nichtregierungsorganisationen las- } \\
\text { sen hoffen. Die deutschen Pneumolo- } \\
\text { gen müssen sich an die Spitze der Anti- } \\
\text { raucher-Bewegung setzen und daher } \\
\text { auch am besten informiert sein. }\end{array}$ & $\begin{array}{l}\text { - Das Risiko durch Passivrauchen an Herzkreislauferkran- } \\
\text { kungen zu erkranken erhöht sich um } 23 \% \text { und das an } \\
\text { Lungenkrebs um } 26 \% \text {. } \\
\text { - Babies, deren Mütter rauchen, erleiden 2mal häufiger den } \\
\text { plötzlichen Kindstod. } \\
\text { - Kinder, deren Eltern rauchen, entwickeln 1,5mal häufiger } \\
\text { Asthma. } \\
\text { - Das Risiko für Kinder rauchender Eltern, an Atemwegser- } \\
\text { krankungen erkranken, liegt } 50 \text { - } 72 \% \text { höher als bei } \\
\text { nichtrauchenden Eltern. } \\
\text { - Zusatzstoffe wie Menthol, Kakao, Lakritz u.v. a. erleich- } \\
\text { tern den Einstieg zum Zigarettenrauchen („milder } \\
\text { Rauch“), erhöhen das Suchtpotenzial (pH-Verschiebung } \\
\text { mit vermehrter Nikotinfreisetzung) und sind potenziell } \\
\text { kanzerogen. } \\
\text { - Nichtraucherschutz seit 10/2002 als } § 3 a \text { in Arbeitsstät- } \\
\text { tenverordnung aufgenommen. }\end{array}$ & $\begin{array}{l}\text { - Doll R, Peto R, Boreham J et al.: } \\
\text { Mortality in relation to smoking: } 50 \text { year's } \\
\text { observation on male British doctors. } \\
\text { BMJ 2004; 328: } 519-533 \\
\text { - Dem Tabakkonsum Einhalt gebieten - } \\
\text { Ärzte in Prävention und Therapie der } \\
\text { Tabakabhängigkeit. } \\
\text { Rote Reihe Tabakprävention und Tabak- } \\
\text { kontrolle. } \\
\text { Deutsches Krebsforschungszentrum Hei- } \\
\text { delberg 2005 } \\
\text { - Dt. Ges. f. Suchtforschung und Suchtthe- } \\
\text { rapie. } \\
\text { Leitlinie Tabakentwöhnung } \\
\text { AWMF-Leitlinien-Nr. 076/006, } 2004\end{array}$ & \\
\hline 2 & Asbestassoziierte Erkrankungen & & & \\
\hline \multirow[t]{2}{*}{2.1} & Epidemiologie & & & 2 \\
\hline & & $\begin{array}{l}\text { - In der Zeit von } 1995 \text { - } 2029 \text { wird sich die Zahl der as- } \\
\text { bestinduzierten Mesotheliomtoten in Europa verdoppeln. } \\
\text { - Erst um das Jahr } 2018 \text { wird der Inzidenzgipfel erreicht } \\
\text { sein; von da an ist mit einem allmählichen Rückgang zu } \\
\text { rechnen. }\end{array}$ & $\begin{array}{l}\text { - Peto J et al.: } \\
\text { European mesothelioma epidemic. } \\
\text { British Journal of Cancer 1999; 79: } \\
666-672\end{array}$ & \\
\hline
\end{tabular}


Die Abgrenzung einer benignen Pleurazellproliferation bei asbest-induziertem Pleuraerguss von Frühmesotheliom oder von metastatischem Karzinom kann schwierig sein.
- Die onkogene Potenz von Fasern hat mit ihrer Länge, ihrer Dicke und ihrer biologischen Beständigkeit im Organismus zu tun.

- Die Kanzerogenität nicht asbesthaltiger Fasern ist in vitro und in vivo geringer, aber prinzipiell bei jeder Faser nachweisbar.

2.3 Klinik

- Pleura-Plaques entgehen dem Röntgennachweis zu 75\%, dem CT immerhin noch in $50 \%$.

sammenhangs einer Lungenerkrankung mit einer früheren Asbestexposition ist die sorgfältige Anamnese

- Die Diagnose der benignen Asbestpleuritis ist per exclusionem zu stellen, erst nach 3-jähriger Nachbeobachtung lässt sich ein Mesotheliom ausschließen.

- Die benigne Asbestpleuritis verläuft oft mit schwerer Defektheilung ab (diffuse Pleurafibrose mit schwerer Restriktion, bis zur respiratorischen Insuffizienz), besonders nach Einnahme des Anti-Parkinsonmittels Bromocriptin.

- Zu Pleuramesotheliom und Bronchialkarzinom, in Zusammenhang mit Asbest siehe dort.
- Müller K-M:

Pleuramesotheliom-Pathologie und Pathogenese.

Pneumologie 2004; 58: 670-679

- American Thoracic Society:

Diagnosis and Initial Management of Nonmalignant. Diseases Related to Asbestos. Am J Respir Crit Care Med 2004; 170: $691-715$

- Konietzko N, Woitowitz H-J, Sohrab S et al.: Das maligne Pleuramesotheliom. Deutsches Ärzteblatt 2000; 97:3257

2.4 Therapie Siehe Kapitel I und L

2.5 Begutachtung

Quantitativ spielen asbestinduzierte pleuropulmonale Berufskrankheiten die wichtigste Rolle in der pneumologischen Begutachtung

3 Pneumokoniosen (außer Asbestosen)

\subsection{Antrakosilikose}

Der Begriff Pneumokoniose umfasst alle nicht neoplastischen Lungenerkrankungen, verursacht durch Inhalation mineralischer oder metallischer Partikel oder durch Staub, ausschließlich Asthma bronchiale, Bronchitis und Emphysem. Ursächlich ist Kohlestaub mit unterschiedlichem Quarzgehalt. Neben der üblichen Kaskade der Fibroseentwicklung (quarzstaubbedingter Zellschaden, Reparaturphase mit Fibrose unter Einfluss verschiedener Zytokine) führen Kohlenstäube zu Bronchitis und Lungenemphysem (siehe unten!).

\subsection{Silikose}

Die Silikose im engeren Sinne wird hervorgerufen durch die Stäube von Sandstein (u. a. wie Quarzit, Grauwacke, Kieselerde, Kieselschiefer, Quarzitschiefer, Granit, Porphyr, Bimsstein, Kieselgur und von keramischen Massen). Als gefährdete Berufsgruppen gelten: Erzbergleute, Schacht- und Gesteinshauer, auch im Steinkohlenbergbau, jedoch nicht Bergleute „vor Ort“.
BK der Nr. 4104 BKV (Lungen- und Kehlkopfkrebs in Verbindung mit Asbestose von Lunge und/oder Pleura oder bei Nachweis der Einwirkung einer kumulativen Asbestfaserstaub-Dosis am Arbeitsplatz von mindestens 25 Faserjahre $(25 \mathrm{~F} / \mathrm{ml} / \mathrm{Jahr}))$.Bei fehlender beruflicher Asbeststaubexposition kann die BK-Anerkennung schwierig sein, da auch Brückenbefunde oder Asbestkörperchennachweis keine berufliche, sondern lediglich umweltbedingte Exposition nachweisen.

- Asbestinduzierte Pleura-Plaques sind Marker der Asbestexposition und daher meldepflichtig, wenn auch nicht entschädigungspflichtig (keine Funktionsausfälle!)

- Andere seltene asbestassoziierte Tumoren (Kehlkopfkrebs, Peritonealmesotheliom, Perikardmesotheliom) sind in die die Liste der Berufskrankheiten aufgenommen.

In Abhängigkeit vom Quarzgehalt des inhalierten Staubes resultieren:

- Simpel CWP = Antrakosilikose (stabil bis langsam progredient) und

- progressive massive Fibrose (PMF) mit frühzeitiger Progredienz.
Kroidl R, Nowak D, Seysen U:

Bewertung und Begutachtung in der Pneumologie 2. Aufl.

Georg Thieme Verlag, Stuttgart 2000.
Merget R, Brüning T:

Entschädigungspraxis gering gestreuter Bergarbeiterpneumokoniosen - neue Berufskrankheiten, neue Konvention oder alles beim Alten lassen?

Pneumologie 2004; 58: 7

- Eibel R, Weber A, Stolpe $S$ et al. Zusammenhang zwischen Emphysemtyp und Schweregrad der Pneumokoniose bei Bergleuten: Korrelation von HRCT-Befunden und ILO-Klassifikation. RöFo 2002; 174: 846-853
- Bei der Silikose gelten als gefährdete Berufsgruppen Erzbergleute, Schacht- und Gesteinshauer, auch im Steinkohlenbergbau, jedoch nicht Bergleute „vor Ort“.

- Bei Silikose (BK der Nr. 4101 BKV) mit Streuung $</=1 / 1$ und obstruktiver Ventilationsstörung, ist diese eher auf eine BK der Nr. 4111 BKV zu beziehen (Stichtag!).

- Bei Streuungsgraden $=\mid>2 / 2$ wird BK der Nr. 4101 BKV die MdE-Höhe bestimmen.
- Hering $\mathrm{K}$ et al:

Die Weiterentwicklung der ILO-Staub-

klassifizierung.

Pneumologie 2003; 57: 576

- Baur X, Köhler D, Voshaar T:

Positionspapier der Deutschen Gesellschaft für Pneumologie zur Begutachtung der Silikose.

Pneumologie 2005; 59: 549-553 
3.3 Chronische obstruktive Bronchitis oder Emphysem der Bergarbeiter

Die chronische obstruktive Bronchitis und/oder das Emphysem von Bergleuten unter Tage im Steinkohlenbergbau (BK der Nr.4111 BKV) bereitet nach wie vor, nicht zuletzt wegen der Stichtagregelung, in der Begutachtung erhebliche Probleme. Voraussetzung!

\subsection{Silikose und Lungenkrebs}

„Lungenkrebs durch Einwirkung von kristallinem Siliziumdioxid $\left(\mathrm{SiO}_{2}\right)$ bei nachgewiesener Quarzstaublungenerkrankung (Silikose oder Siliko-Tbc)“ lautet die Bezeichnung für die zukünftige BK der Nr. 4112 BKV.

\subsection{Seltene Pneumokoniosen}

Neben einer Reihe von exotischen Pneumokoniosen wie der Baritose, der Siderose, der Stenose oder der Aluminiumlunge sind die Berilliose und die Schweißerlungenfibrose in den letzten Jahren in den Fokus der Arbeitsmedizin gerückt.
- Nachweis der Einwirkung einer kumulativen Feinstaubdosis von i. d. R. 100 (mg/m $\mathrm{m}^{3}$ Jahre).

- Veränderungen im Thorax-Röntgenbild sind keine

- Stichtagsregelung (keine BK-Anerkennung, wenn Versicherungsfall vor 01.01.1993 eintrat).

- Außer Nachweis einer obstruktiven Ventilationsstörung kann auch der alleinige Nachweis einer relevanten unspezifischen bronchialen Hyperreagibilität eine BK begründen.

- Eine relevante und langfristig reproduzierbare Lungenüberblähung kann als Emphysemzeichen gelten.

- Ein durch HRCT belegtes Lungenemphysem begründet einen Versicherungsfall auch ohne Lungenfunktionsstörung.

- Bis zur Novellierung Anerkennung nach der Öffnungsklausel (§9, Abs. 2 SGB VII).

- Gefährdete Berufsgruppen (Erzbergleute, Schacht- und Gesteinshauer, auch im Kohlebergbau, jedoch nicht Bergleute „vor Ort“, Tunnelarbeiter, Gussputzer, Sandstrahler, Ofenmaurer, Former in der Metallindustrie, Gießereiarbeiter und viele andere mehr.

- Die Diagnosen „Lungenkrebs“ und „Silikose“ müssen gesichert sein, um eine BK der Nr. 4112 anzuerkennen.

- Uneinigkeit besteht bei alleiniger Hilus-LymphknotenSilikose, bei kleinen rundlichen Schatten $=/ 1 / 1$ nach ILO und bei computertomographischer Nachweis kleiner Herdschatten ohne Auffälligkeit in der Rö-Thorax-Übersicht.

- „Schweißerlungenfibrosen“ als Berufskrankheit beschäftigt derzeit (02/05) den Sachverständigenausschuss beim BMAW.

- Die Berylliose ist wegen gehäuftem Vorkommen (Chipherstellung) und verbesserter Diagnostik (BAL) aktuell.

- Klinisch ist die Berylliose nicht von der Sarkoidose zu unterscheiden.

- Bauer X

Auswirkungen der untertägigen Belastungen im Steinkohlenbergbau auf die Lunge. Pneumologie 2004; 58: 107-115

- Baur X, Köhler D, Voshaar T: Positionspapier der Deutschen Gesellschaft für Pneumologie zur Begutachtung der Silikose.

Pneumologie 2005; 59: 549-553

\section{Medikamenteninduzierte Lungenschäden}

Die Vielfalt der pulmonalen Reaktionen und die große Zahl der potenziell pulmotoxischen Substanzen erschweren die Diagnose erheblich. „Darandenken“ und eine penible Medikamentenanamnese können vor verhängnisvollen Fehldiagnosen schützen.

- Atemwege (Bronchospasmus durch Beta-Blocker, Husten bei ACE-Hemmern).

- Lungenparenchym (zytotoxisch wirkend, nicht zytotoxisch wirkend: Amiodaron, Furantoin F, Sulfasalazine und andere).

- Medikamentös induzierter LE.

- Eosinophile Lungenerkrankungen durch Medikamente.

- Lungengefäßbeteiligung (Aminorex, Kontrazeptiva, iv-Injektion von Drogen).

- Pleura-/Perikarderguss (Bromocriptin).

- Atemmuskulatur (Aminoglykoside).

- Mediastinum (M. Ormond bei Methergin, Lipomatose bei iatrogenem Cushing).

- Zytokininduzierte Lungenerkrankungen.

- Alveoläres Hämorrhagie-Syndrom.

5. Strahleninduzierte Lungenerkrankungen

Neue Modalitäten in der modernen Strahlentherapie und der Einsatz von Radiosensitizern haben Häufigkeit und Erscheinungsbild der Strahlenreaktionen der Lunge verändert.
- Strahlenpneumonitis als Akutschaden: Risikofaktoren, Prävention!!

- Strahlenfibrose als Spätfolge: Latenz von Wochen bis Monaten.

- Radio-Recall-Effekt nach früherer Strahlentherapie und konsekutiver Zytostatikagabe.

- Radiosensitizer (Zytostatika, $\mathrm{O}_{2}$-Applikation).
- Kroidl R, Nowak D, Seysen U: Bewertung und Begutachtung in der Pneumologie 2. Aufl. Georg Thieme Verlag, Stuttgart 2000

- Buerke U, Schneider J, Müller KM et al. Schweißerlungenfibrose: Begründung für die Aufnahme als neue Berufskrankheit. Pneumologie 203; 57: 9-14

- Eine aktuelle Liste aller potenziell lungentoxischer Medikamente kann unter http://www.pneumotox.com. abgerufen werden.

- Camus P et al.:

Drug induced infiltrative lung disease. Eur Respir ] Suppl. 2001; 32: 93s-100s cancer: sobering results. J Clin Oncol 2001; 1 (9): 1734-1742 Breathnach OS, Freidlin B, Conley B et al.:
Twenty-two years of phase III trials for pa- 
6. Toxische Lungenschädigungen

6.1 Akut

Hohe Schadstoffkonzentrationen verur- - Chlorgas (Schwimmbäder!)

sachen im Allgemeinen reparable Akut- - Formaldehyd (Nasenkrebs nur bei chronischer Exposischäden oder Tod, wiederholte, niedrige Irreparable Dauerschäden. Gut wasserlösliche Gase schädigen mehr die oberen Atemwege (z. B. $\mathrm{SO}_{2}>$ Bronchitis), schlecht wasserlösliche die Alveolen (z. B. Lungenödem durch Chlorgas). tion)

- Ammoniak (Zusatzstoff im Tabak!)

- Isozyanate (Dauerschäden bei intermittierender Inhalation!)

- Lost (Gaskriegsopfer im Irak/Iran-Krieg)
- Feuerrauch („,burnt lung“)

\subsection{Chronische Schäden der Atemwege}

Nach den Formalin- und Ozondebatten machen derzeit Feinstaub, Radon und Passivrauchen Schlagzeilen. Der Pneumologe muss informiert sein, denn sein Fachwissen ist gefragt!
- Feinstaub (Definition, kardiovaskuläre Risiken bei akuter Belastung, Krebsrisiko, Anteil Dieselruß nur 20\%).

- Luftverschmutzung („London-Typ“, „Los Angeles-Typ“)

- Luftverunreinigung in Innenräumen (Indoor-air-pollution).

- Passives Tabakrauchen.

- Emission bei offenen Feuerstellen $\left(\mathrm{CO}_{2}\right)$.

- Energiereiche $\mathrm{O}_{2}$-Radikale wie NOX.

- Organische Lösungsmittel, Baumaterialien.
- Voshaar TH, Heyder J, Köhler D et al.: Partikelbelastung und menschliche Gesundheit - Was ist zu tun? Pneumologie 2005; 59: 470-474

- AWMF-Leitlinie 2001 Deutsche Gesellschaft für Arbeitsmedizin und Umweltmedizin e. V. (DGAUM) Arbeitsmedizinische Vorsorgeuntersuchungen bei Belastung durch atembaren alveolengängigen Staub (A-Staub).

\section{Tauchkrankheiten}

Bei der Überwachung von Caisson-

- Ertrinken, „Beinahe-Ertrinken“ und „trockenes Ertrinken“. arbeitern, insbesondere im Tunnel- und - Salzwasser-/Süßwasser-Aspiration.

U-Bahn-Bau, und bei Überprüfung der Tauchtauglichkeit muss der Pneumologe Spezialkenntnisse besitzen.

- Tauchunfälle bei Apnoetauchern, Schnorchlern und Gerätetauchern

- Caissonarbeiten mit den Gefahren des Barotraumas und des Pneumothorax.

Medizinische Tauchfibel.
- Verordnung zum Schutz vor gefährlichen Stoffen (GefahrstoffverordnungGefStoff V). 16. Aufl.

Carl Heymann Verlag 2001

8. Höhenkrankheit

Beratung und Eignungsuntersuchungen bei Flugzeugfliegen (ca. 2500 Höhe entsprechend) und Hochgebirgstourismus (bis zu Siebentausendern) sind angewandte Höhenphysiologie.
Akute Höhenkrankheit mit Lungenödem und Hirnödem: Pathogene, Therapie und Prävention.

- Chronische Höhenkrankheit mit Cor pulmonale.

- Respiratorische und hämodynamische Komplikationen bei Lungenpatienten in der Höhe (inklusive Flug).

\section{Atemwegserkrankungen}

Asthma bronchiale

1.1 Definition

Neu bei der Definition ist die als ursächliche angesehen Entzündung der Atemwege.
Die derzeitige Definition beinhaltet neben der bronchialen Hyperreagibilität und der variablen Atemwegsobstruktion den Terminus der Atemwegsentzündung.

\section{$1.2 \quad$ Epidemiologie}

Die Epidemiologie des Asthma zeigt in den letzten 10 Jahren erfreuliche Trends.
- Die Asthma-Inzidenz nimmt in den letzten 2 Jahrzehnten weltweit leicht ab.

- In Deutschland nahmen im letzten Jahrzehnt die direkten Gesundheitskosten pro Fall graduell ab.

- Die Asthmamortalität in Deutschland geht ebenfalls zurück.

- Die Asthma-Inzidenz nimmt in den letzten 2 Jahrzehnten weltweit leicht ab.

- In Deutschland nahmen im letzten Jahrzehnt die direkten Gesundheitskosten pro Fall graduell ab.

- Die Asthmamortalität in Deutschland geht ebenfalls zurück.
- Matthys $\mathrm{H}$

Springer-Verlag, Berlin 1983.

- Leitlinie der Gesellschaft für Tauch- und Überdruckmedizin.

Leitlinie Tauchunfall

1 WMF 2005

Mechanisms Of Disease: Pulmonary Arterial Hypertension.

N Eng J Med 2004; 351: 1655- 1665

\subsection{Pathogenese}

Aufgrund der immensen Neuerkenntnisse in der Molekular- und Zellbiologie erwarten wir gespannt, wie dieses Wissen auch praktisch in der Diagnostik und Therapie umgesetzt wird.
- Von den Zellbiologen wurden in den letzten Jahrzehnten die Akteure der asthmatischen Entzündung (Alveolarmakrophage, Eosinophile, TH2-Lymphozyt) wohl definiert.

- Die Molekularbiologie hat die immunologischen Abläufe, insbesondere bei der exogen-allergischen Form, weitgehend aufgeklärt.
- Deutsche Atemwegsliga und Deutsche Gesellschaft für Pneumologie:

Leitlinie zur Diagnostik und Therapie von Asthma.

Kurzfassung, Georg Thieme Verlag, Stuttgart 2005

Fabel H, Konietzko N:

Weißbuch Lunge 2005.

Thieme-Verlag, Stuttgart 2005

- European Lung White Book

European Respiratory Society and

European Lung Foundation 2003

- Leitlinie der Deutschen Gesellschaft für Kardiologie -Herz- und Kreislaufforschung:

Diagnose und Behandlung der chronischen koronaren Herzerkrankung.

AWMF-Leitlinien-Register Nr. 019/001, 2003 
1.4 Diagnostik

In den neuen Leitlinien hat die Objektivierung der Atemwegsobstruktion durch die Lungenfunktionsprüfung, ihre Reversibilität nach Bronchodilatatoren und die Reaktion auf Histamin einen noch höheren Stellenwert für die Diagnose als bisher erhalten.
- Die neuen Leitlinien sehen einen diagnostischen Algorithmus mit standardisierter Definition von Reversibilität nach Bronchodilatanzien vor:

- Nachweis einer Obstruktion $\left(\mathrm{FEV}_{1} / \mathrm{VK}<70 \%\right)$ und $\mathrm{FEV}_{1}$-Zunahme $>15 \%$ (mindestens $200 \mathrm{ml}$ ), bezogen auf den Ausgangswert.

- Differenzialdiagnostisch ist am wichtigsten die Abgrenzung gegenüber der COPD.

- Differenzialdiagnostisch sind aber auch die gastroösophageale Refluxkrankheit und Stimmbanddysfunktionen mit einzubeziehen.

\subsection{Schweregrad und Verlauf}

Die Schweregradeinteilung aufgrund der Symptomatik und der Lungenfunktion ist die Basis für den Therapieplan. Eine besondere Herausforderung für den Pneumologen ist die Betreuung von Asthmatikerinnen in der Schwangerschaft.
- Der praktische Wert der PEF-Messung liegt in der Erfassung der zirkadianen Variabilität der Atemwegsobstruktion (PEF morgens $<20 \%$ niedriger als PEF abends; PEFVariabilität $=$ PEFmo-PEFab $/$ PEFmo).

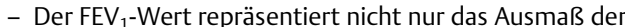
bronchialen Obstruktion, sondern ist auch der wichtigste Parameter zur Beurteilung des natürlichen Krankheitsverlaufs sowie des Ansprechens auf eine Bronchodilatator-Therapie.

- Bei asthmatischen Frauen besteht ein erhöhtes Risiko fü Präeklampsie bei der Schwangeren und Frühgeburt sowie niedrigem Geburtsgewicht beim Kind.
- Menz G et al.:

Schwieriges Asthma: Klinische Phänotypen und Prinzipien der Therapie.

Der Pneumologe 2004; 1: 9-15

- Ahrens P, Seibt Y, Kitz R:

Vocal Cord Dysfunction bei Kindern und Jugendlichen.

Pneumologie 2001; 55: 378-384

- Kardos P:

Neue Konzepte in der Therapie des Asthma bronchiale.

Der Pneumologe 2004; 1: 16-23

- Deutsche Atemwegsliga und Deutsche Gesellschaft für Pneumologie: Leitlinie zur Diagnostik und Therapie von Asthma.

Pneumologie 2005; 59: $x-y$

- Murphey VE, Gibson PG, Smith R, Clifton $\mathrm{VL}$ :

Asthma during pregnancy: mechanisms and treatment implications.

Eur Respir 」 2005; 25: 731 - 750

1.6 Therapie
Die Asthma-Therapie gehört zu den Erfolgsstories der modernen Medizin. Entscheidend für ein erfolgreiches Asthmamanagement sind die Schulung des Patienten, die konsequente Umsetzung der Maßnahmen, insbesondere der Anwendung der Dauermedikation, und die regelmäßige Kontrolle.

- Ziel der medikamentösen Therapie ist die Unterdrückung der asthmatischen Entzündung und damit sekundär der bronchialen Hyperreagibilität und der Atemwegsobstruktion.

- Die Asthmamedikamente werden unterteilt in Controller (Dauermedikation zur Langzeittherapie) und Releaver (Bedarfsmedikation).

- Neu sind weniger die Medikamente, als vielmehr ihre stringente Anwendung in einem Stufenplan, der sich nach dem augenblicklichen Schweregrad der Erkrankung richtet.

- Die Behandlung beginnt auf der Stufe, die dem augenblicklichen Schweregrad der Erkrankung entspricht. Falls keine adäquate Kontrolle erreicht wird, erfolgt der Übergang auf die nächsthöhere Stufe (Step up-Prinzip).

- Die Inhalationssysteme sind in den letzten Jahren erheblich verbessert worden und erlauben nicht nur eine immer präziserer Applikation der Dosis, sondern auch eine gezieltere Applikation auf verschiedenen Ebenen der Atemwege (Targeting)

- In der Entwicklung gezielterer Asthmamedikamente sind Fortschritte zu verzeichnen: Anti-lgE-Antikörper, Leukotrienrezeptorantagonisten.

- Nichtmedikamentöse Maßnahmen (Patientenschulung, körperliches Training, Tabakentwöhnung, psychosoziale Konzepte, Reduktion des Übergewichtes) sind nicht zu vernachlässigen.

- Bei den meisten nichtschulmedizinischen Therapiemodalitäten ist die Wirksamkeit unbewiesen: Akupunktur, „Alexander Technik“, progressive Relaxation nach Jacobson; Hypnotherapie; autogenes Training; BiofeedbackTraining; Transzendentale Meditation, Buteyko Atemtechnik, Chiropraxis, Ernährungsmaßnahmen (Fischöl, Fettsäuren, Mineralstoffe, Vitamin C), Hypnose, Homöopathie, Ionisierer (Raumluftreiniger), Kontrolle der Luftfeuchtigkeit, Phytotherapeutika, Speleotherapie, Traditionelle Chinesische Medizin.

\subsection{Prognose}

Verlauf und Prognose des Asthma haben sich im letzten Jahrzehnt verbessert. Da aber immer noch Asthmatodesfälle vorkommen, gilt es, Risikopatienten zu identifizieren und engmaschig zu betreuen.
Prognostische Faktoren auf ein erhöhtes Mortalitätsrisiko im Asthmaanfall sind:

- Vorgeschichte mit vorheriger Intubation und Beatmung.

- laufende systemische Steroidtherapie,

- unzureichende topische Steroidtherapie und übermäßiger Einsatz von $\beta_{2}$-Agonisten,
- Gaga M, Siafakas NM:

Special considerations in asthma.

ERS Monograph Asthma 8: 293-311

- Deutsche Atemwegsliga und Deutsche Gesellschaft für Pneumologie:

Leitlinie zur Diagnostik und Therapie von Asthma.

Kurzfassung, Georg Thieme Verlag, Stuttgart 2005

- British Thoracic Society:

British Guideline on the management of asthma.

Thorax 2003; 58 (Suppl.): i1 - i54

- psychosoziale Probleme. 
Obwohl bereits mehrere „Asthma-Gene" identifiziert sind, ist die genetische Reparatur der Krankheit utopisch, es bleibt bei den klassischen Instrumenten der Prävention.

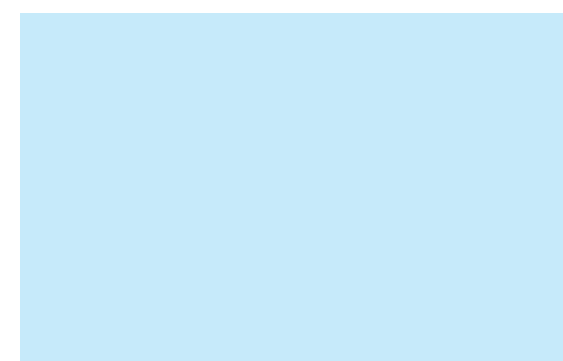

1.9 Begutachtung

Hinweise auf die berufliche Verursachung einer Atemwegserkrankung ergeben arbeitsplatzbezogene Beschwerden und Berücksichtigung der Latenzzeit einer allergischen Sensibilisierung mit Schwankungsbreite von Wochen bi Jahren. Berufsallergene sind oft niedermolekulare Stoffe (Haptene), die erst nach Eiweißbindung im Körper einen antigenen Hapten-Eiweiß-Komplex bilden. Oft schwieriger kutaner und serologischer Sensibilisierungsnachweis.

\section{COPD}

Primärprävention: Stillen zumindest bis zum 6. Monat bei atopischer Belastung ist als effektive Maßnahme erwiesen.

- Passives Mitrauchen ist bei Kindern anfallsfördernd und mit dauerhafter Verschlechterung der Lungenfunktion verbunden.

- Sekundärprävention: Die gängigen Maßnahmen zur Allergenkarenz bei Hausstaubmilben und die Rolle von Akkaroziden sind umstritten.

- Tertiärprävention: Influenzavirus- und Pneumokokkenschutzimpfung umstritten.

- Bei der spezifischen Immuntherapie ist die sublinguale Applikation mangels aussagekräftiger Studienergebnisse zweifelhaft.

- Asthma kann die physischen, emotionalen und sozialen Aspekte im Lebens von Patienten und Angehörigen nachheitlich beeinflussen.

- Die Erfassung Lebensqualität ist daher ein wichtiger Bestandteil des Asthmamanagement.

- Indikationen zur Rehabilitation: Persistierende asthmatische Beschwerden, Gefährdung der Berufs- und Erwerbsfähigkeit, drohende Pflege- und Hilfsbedürftigkeit.

- Isocyanat-Asthma (BK 1315) und durch allergisierende Stoffe verursachte obstruktive Atemwegserkrankungen (BK 4301) müssen „zur Unterlassung aller Tätigkeiten gezwungen haben, die für die Entstehung, die Verschlimmerung und das Wiederaufleben der Krankheit ursächlich waren oder sein können“.

- MdE-Schätzung frühestens 26 Wochen nach Eintritt des Versicherungsfalles ( = Tag der Aufgabe der gefährdenden Tätigkeit).

- Ein negativer arbeitsplatzbezogener bronchialer Provokationstest schließt ein berufsbedingtes Asthma bronchiale nicht aus, weil die bronchiale Obstruktion auf eine andere Allergenverbindung (z. B. Isocyanate) zurückgehen kann. (Falsch negative Provokationstest).
- British Thoracic Society:

British Guideline on the management of asthma.

Thorax 2003; 58 (Suppl.): i1 - i54

\subsection{Definition und Epidemiologie}

Unterschiedliche Definitionen (COPD = chronisch obstruktive Bronchitis + Lungenemphysem!?) und Unschärfen in der Abgrenzung zum Asthma bronchiale ( Schnittmenge?) erschweren die Diagnostik (Eisbergphänomen). Auch der Begriff „akute Exazerbation der COPD“ (AECOPD) wird höchst unterschiedlich verstanden.
- Die Vielzahl der verschiedenen Definitionen der COPD (ATS, BTS, DGP/Deutsche Atemwegsliga, GOLD, ERS) ist verwirrend, wird aber derzeit international abgestimmt. Es empfiehlt sich, die Definition der DGP/Deutsche Atemwegsliga zu benutzen.

- Die Bedeutung der COPD als Volkskrankheit ist erst in den letzten Jahren klar geworden, die Zunahme von Inzidenz und Prävalenz weltweit lässt sie als dritthäufigste Todesursache nach den kardiovaskulären Erkrankungen ab 2010 prognostizieren.

- Entsprechend groß sind die direkten (Arzt, Krankenhaus, Pharmaka) wie die indirekten (EU, AU, Verlust an Arbeitskraft) Kosten für das Gesundheitswesen.

- Bei der AECOPD sind die klassischen Anthonisen-Kriterien (Zunahme von Atemnot + Sputumvolumen, Sputumverfärbung) als Prädiktoren einer erfolgreichen Antibiotikatherapie wenig geeignet: $80 \%$ der Patienten werden unnötig behandelt.

- Raucher mit COPD haben ein größeres kardiovaskuläres Risiko als Raucher ohne COPD.
- Kroidl R, Nowak D, Seysen U:

Bewertung und Begutachtung in der Pneumologie 2. Aufl.

Georg Thieme Verlag, Stuttgart 2000 


\section{2 Ätiologie und Pathogenese}

Die Zellbiologie und die Molekulargenetik haben klar die Bedeutung der bronchialen und alveolären Entzündung für Umbau der Atemwege (Remodelling) und die Destruktion der Alveolen herausgestellt. Darüber hinaus wird mehr und mehr klar, dass es sich bei der COPD nicht um eine Systemerkrankung mit kardiovaskulären, muskuloskelatalen, metabolischen und zentralnervösen Manifestationen handelt.

\subsection{Diagnostik}

Die Lungenfunktionsdiagnostik (Obstruktionsparameter, Reversibilität, Emphysemkonstellation) mit Anamnese und klinischer Befund sind entscheidend für die COPD-Diagnose. Bildgebende Verfahren (Rö-Thorax, CT) und Laborbefunde (Alpha-1-Antitrypsin) dienen der differenzialdiagnostischen Abgrenzung.
- Da die COPD als Summe von chronisch obstruktiver Bronchitis und Lungenemphysem definiert ist, nimmt es nicht Wunder, dass die Pathogenese auf den beiden unterschiedlichen Ebenen des Respirationstrakts auch unterschiedlich ist.

- Der gemeinsame pathogenetische Faktor ist das Tabakrauchen. Diese inhalalierten Schadstoffe (Gase, Partikel) führen in der Bronchialschleimhaut zu einer granulozytären Entzündung mit Zerstörung der zilientragenden Zellen, Infekten infolge mukoziliarer Insuffizienz und Umbau der Schleimhaut. Die Folge ist eine Dauerobstruktion mit geringer Reversibilität.

- Zunehmend wird die COPD als Systemerkrankung erkannt. Auch die Pathogenes der nicht-pulmonalen Organmanifestationen, insbesondere im Herzkreislaufsystem, wird besser verstanden.

- In den Alveolen kommt es zur Anhäufung von Alveolarmakrophagen und Granulozyten. Beide setzen Elastasen und Oxidanzien frei, deren Gegenspieler, die Antielastasen und Antioxydantien, durch den Tabakrauch inaktiviert werden. Die Folge ist die Zerstörung des Lungengewebes (=Emphysem).

- Entsprechend der Definition als chronisch obstruktive Lungenerkrankung steht im Zentrum der Diagnostik die Lungenfunktionsdiagnostik (Obstruktionsparameter, Reversibilität, Emphysemdiagnostik)

- Die Lungenfunktion ist auch für die Verlaufskontrolle und für die Schweregradfeststellung entscheidend.

- Die Frage der Frühdiagnostik durch die quantitative Computertomographie ist derzeit noch im Fluss, dank dieser Methode ist jedoch der Stellenwert von weniger aufwändigen Tests (z. B. der Diffusionskapazität) besser zu evaluieren.

- Generell sind weniger die diagnostischen Tests neu, als vielmehr deren synoptische Deutung bei der Diagnose und Differenzialdiagnose der COPD.

\subsection{Verlauf und Schweregrad}

\subsection{Therapie}

Die Behandlung der COPD beinhaltet die medikamentöse und nichtmedikamentöse Dauertherapie und die Intervention bei akuter Exazerbation.
- Eine Reihe neuer Erkenntnisse über den Verlauf der COPD konnte aus den großen internationalen Therapiestudien gewonnen werden. Die entscheidende Maßnahme ist die Tabakabstinenz, nur sie vermag eine weitere Progredienz der Erkrankung zu verhindern.

- Über die Definition der Schweregrade gibt es leider international keinen Konsens, es empfiehlt sich jedoch die deutsche Schweregradeinteilung (nach klinischen und Lungenfunktionskriterien) auch im Hinblick auf die Therapie und ökonomische Konsequenzen zu übernehmen.

- Die Progredienz der COPD lässt sich bei ihrem langen Verlauf auch im Einzelfall gut mit der jährlichen Abnahme des FEV ${ }_{1}\left(-\mathrm{FEV}_{1}\right)$ beschreiben. Regelmäßige spirometrische Messungen präzisieren die Aussage.

- Die Behandlung der COPD erfolgt nach der DGP-Leitlinie stadiengerecht.

- Bei der medikamentösen Therapie werden den langwirksamen Betamimetika und/oder Anticholinergika, obwohl teurer, wegen der besseren Patientencompliance der Vorzug gegeben.

- Inhalative Glukokortikoide sind bei Schweregrad II und III nur indiziert, wenn der Ausgangs-FEV $>15 \%$ ansteigt oder die Symptomatik sich innert 3 Monaten bessert.

- Systemische Glukokortikoide werden nur bei Exazerbation der COPD empfohlen.

- Die nichtmedikamentöse Therapie (körperliches Training, Physiotherapie, Patientenschulung und Ernährungsberatung, Langzeitsauerstofftherapie, nicht invasive Beatmung, Emphysemchirurgie) spielt wegen der geringen Wirksamkeit der Pharmakotherapie eine besondere Rolle.
- Farmer SG, Rennard SI, Voelkel N: Chronic obstructive pulmonary disease: A Disorder of the Cardiovascular and Respiratory Systems.

Proc Thorac Soc 2005; 2: 7- 100
- Kauczor M, Markstaller K, Heussel CP:
Pulmonale Struktur und Funktion
Hochauflösende und dynamische Compu-
tertomographie bei Lungenemphysem
und Erkrankungen der Atemwege.
Pneumologie 2002; 56: 24-30

- Becker E et al.

Leitlinien zur sozialmedizinischen Leistungsbeurteilung bei chronisch obstruktiven Lungenkrankheiten (COPD) und Asthma bronchiale.

Gesundheitswesen 2004; 66: 439-456

- ATS/ERS: Standards for the Diagnosis and Management of Patients with COPD (2005).

www.thorax.org/copd

- Worth H, Kardos P, Magnussen $\mathrm{H}$ et al.: Leitlinie der Deutschen Atemwegsliga und der Deutschen Gesellschaft für Pneumologie zur Diagnostik und Therapie von $\mathrm{Pa}$ tienten mit chronisch obstruktiver Bronchitis und Lungenemphysem (COPD). Pneumologie 2002; 56: 704-738

- Barnes PJ, Stockley RA: COPD: current therapeutic interventions and future approaches. Eur Respir J 2005; 25: 1084-1106

- Gillissen A et al.: Management der akuten Exazerbation der chronisch-obstruktiven Lungenkrankheit. DMW 2003; 128: 1721-1727 


\subsection{Rehabilitation}

Rehabilitationsprogramme zeigen bei der Besserung der Lebensqualität und der Belastungstoleranz bessere Ergebnisse als alle anderen Maßnahmen.
- Rehabilitationsmaßnahmen steigern die AusdauerBelastbarkeit und senken die Dyspnoeschwelle.

- Rehabilitationsmaßnahmen sind in allen Stadien der Erkrankung effektiv.

- Troosters T, Casaburi R, Gosselink R, Decramer M:

Pulmonary Rehabilitation in Chronic Obstructive Pulmonary Disease. Am J Respir Crit Care Med 2005; 172: $19-38$

2.7 Prävention

Die Vermeidung von Risikofaktoren, zuvorderst des Tabakrauchs, steht im Zentrum der Prävention. Physische Rekonditionierung steigert die Lebensqualität, Impfungen reduzieren Schwere und Zahl der akuten Exazerbationen bei COPD. - Durch Reduktion der Dyspnoe können die Lebensqualität
des Patienten und die häufig assoziierte Depression gebessert werden.

- Entscheidend für den nachhaltigen Erfolg dieser Maßnahmen ist die nachgehende ambulante Kontrolle unter Einsatz der Lungenfunktion.

- Grippe- und Pneumokokken-Impfungen werden bei allen COPD-Patienten empfohlen, obwohl die Evidenz nicht eindeutig ist.

\subsection{Begutachtung}

Als Sonderfall der COPD ist die chronische obstruktive Bronchitis oder Emphysem von Bergleute zu beachten (BK der Nr. 4111 BKV).

Siehe auch Kapitel E.

- Bundesärztekammer in Zusammenarbeit mit der Kassenärztlichen Bundesvereinigung. Frei von Tabak - Ein Stufenprogramm zur Raucherberatung und Rauchertherapie in der Arztpraxis (3. überarbeitete Auflage) Deutscher Ärzteverlag Köln. 2001

- Batra A, Buchkremer G:

Tabakabhängigkeit - Ein Therapeutenleitfaden.

Kohlhammer, Stuttgart, 2003

- Chronische obstruktive Bronchitis oder Emphysem von Bergleuten unter Tage im Steinkohlenbergbau bei

- Nachweis der Einwirkung einer kumulativen Feinstaubdosis von i.d. R. 100 ( $\mathrm{mg} / \mathrm{m}^{3} \times$ Jahre).

3 Bronchiektasen

3.1 Definition und Epidemiologie

Die Definition einer irreversiblen Dilatation ab Segmentbronchusebene gilt unverändert, die Einteilung erfolgt nach Klinik (trocken/feucht), Pathogenese (angeboren/erworben) und Adiologie (zylindrisch/sarkulär).

\section{2 Ätiologie und Pathogenese}

Der Pathomechanismus, der zur Ausbildung von Bronchiektasen führt, ist der Knorpeldefekt, selten angeboren, meist erworben (Infektion!).
Bei den erworbenen Bronchiektasen stehen die generalisierten Bronchiektasen bei Mukoviszidose und primärer ziliarer Dyskinesie im Vordergrund.

- Durch die hohe Treffsicherheit des HRCT's in der Diagnostik haben Inzidenz und Prävalenz (nur scheinbar?) zugenommen.

\subsection{Verlauf und Schweregrad}

Der Nachweis von Bronchiektasen ist nicht gleichbedeutend mit „Bronchiektasen-Krankheit", die Verläufe daher ganz unterschiedlich.
- Die Diagnostik der erworbenen Bronchiektasen ist seit der Einführung des Schweißtests (Mukoviszidose!), der Ziliarkinetik und des NO-Exhalates ( $P C D=$ Primäre ziliare Dyskinesie) und der Immunglobuline (IgA, IgG, IgG-Subklassen) auf sicherem Boden.

- Die Stellung des nasalen NO-Exhalats in der Diagnostik ist noch umstritten.

- Zur ABPA siehe Kapitel N.

- Bei den bildgebenden Verfahren hat sich das HRCT eindeutig gegenüber der Bronchographie und dem ordinären $C T$ durchgesetzt.

M Puderbach et al:

Visualisierung von Lungenparenchymveränderungen bei Patienten mit Zystischer Fibrose - MRT versus HRCT.

Röfo 2004; 176

- Felix H, Holzmann D:

Funktion und Ultrastruktur der Zilien be der primären ziliaren Dyskinesie.

Schweizer Med Wochenschr 2000; 130: 699-704

- Reinhardt D, Götz M, Kraemer R. Schöni MH (Hrsg):

Zystische Fibrose.

Springer, Heidelberg 2001

- Hering KG, Rodenwaldt J:

Bildgebende Verfahren in der Pneumologie: Atemwege.

Der Pneumologe 2005; 2: 393 - 406

- Wodehouse T et al.:

Nasal nitric oxide measurement for screening of primary ciliary dyskinesia. Eur Respir ] 2003; 21: 43-47

- Verlauf und Schweregrad der Bronchiektasen-Krankheit hängen wesentlich ab von der Grunderkrankung (CF, ABPA, PCD, Immundefekt) und der Zahl und Lage der befallenen Segmente.

- Die Kolonisierung/Infektion mit Staphylokokken und Pseudomonas verschlechtert die Prognose.

- Pulmonale Mycobacterium-avium-Infektion kann bei nicht-immungeschwächten Patienten zu Bronchiektasen führen, umgekehrt können Bronchiektasen sekundär mit (meist atypischen) Mykobakterien besiedelt werden.
- Konietzko N:

Pulmonale Mycobacterium-avium-Infektion bei nicht-immungeschwächten Patienten: Eine neue Krankheit? Pneumologie 2000; 54: 454

- Hering KG, Rodenwaldt J: Bildgebende Verfahren in der Pneumologie: Atemwege.

Der Pneumologe 2005; 2: 393 - 406 
Unter konsequenter Therapie und Nutzung der präventiven Möglichkeiten können Patienten mit Bronchiektasen heute alt werden.
- Die präventive Inhalation mit Antibiotika (Gentamycin, Tobramycin) kann, wie bei der Mukoviszidose, die Zah der Exazerbationen vermindern und die Progredienz der Erkrankung günstig beeinflussen.

- Chirurgische Maßnahmen sind nur bei Befall von maxima zwei Lappen und nach Ausschluss einer Systemerkrankung sinnvoll.
- Kohlhäufl K, Scheuch G, Häußinger K: Antimikrobielle Aerosoltherapie bei Bronchiektasen?

Pneumologie 2004; 58: 499-504

- Schaberg T:

Inhalative antimikrobielle Therapie bei Bronchiektasen.

Pneumologie 2004; 58: 477

\subsection{Prävention}

Die regelmäßig durchgeführte Atemphysiotherapie mit dem Ziel der Sekretmobilisierung reduziert die Zahl und Schwere der Exazerbationen und entscheidet letztlich über die Prognose des Patienten.

- Atemphysiotherapeutische Maßnahmen mit erwiesener Wirksamkeit beinhalten spezielle Hustentechniken, manuelle Hilfen zur Sekretmobilisierung, Einsatz von Hilfsmitteln wie Flutter, Cornet, PEP-Maske.

- Die Impfprophylaxe gegen Influenza und Pneumokokkus wird empfohlen, auch wenn nicht evidenzbasiert.

- Die 3-monatige iv-Antibiose im krankheitsfreien Intervall ist in ihrer Wirksamkeit nur bei CF erwiesen.

- Wewel AR, Jörres RA, Kirsten D: Möglichkeiten und Perspektiven häuslichen Trainings bei Patienten mit chronisch-obstruktiven Atemwegserkrankungen.

Pneumologie 2005; 59: 328-336

G Generalisierte Lungenparenchymerkrankungen

1 Exogen-allergische Alveolitis (EAA)

1.1 Ätiologie und Epidemiologie

Der Katalog der verursachenden Allergene wird laufend erweitert.
Die neueste Entität ist die „Kornkäferlunge“. Sie entsteht im - Sennekamp H-J:

Viehstall bei Verfütterung von Korn, das vom Kornkäfer befallen ist.
Exogen allergische Alveolitis.

Dustri-Verlag, 2004

1.2 Pathologie

$\begin{array}{lll}\text { Ein spezifisches pathologisch-anatomi- } & \text { Morphologische Hinweise auf das Vorliegen einer EAA sind: } & - \text { Sennekamp H-J: } \\ \text { sches Substrat für die EAA existiert } & \text { - herdförmige lymphozytäre Infiltrate im Interstitium. } & \text { Exogen allergische }\end{array}$

nicht. Der BAL-Befund ist dagegen recht - Bronchiolitis obliterans mit BOOP.

Exogen allergische Alveolitis.

typisch, wenn auch allein nicht diag- - Granulome in uncharakteristischer Verteilung.

Dustri-Verlag, 2004 nostisch.

1.3 Diagnostik

Typisch für die EAA ist die Diskrepanz zwischen deutlichen subjektiven Beschwerden (Husten, Atemnot, Fieber) und deutlicher Lungenfunktionsdefekte auf der einen und einem diskreten Röntgenbefund auf der anderen Seite.
- Das HR-CT mit dem typischen Bild der „Milchglastrübung“ ist ziemlich typisch, wenn auch nicht krankheitsspezifisch.

- Die BAL zeigt im akuten Stadium das Bild einer CD8-Alveolitis.

- Tuengerthal S:

HRCT der exogen-allergischen Alveolitis (EAA).

Allergologie 2001; 24: 136

- Teschler H:

Bildgebende Verfahren in der Pneumologie: Das Lungenparenchym. Der Pneumologe 2005; 2: 407 - 421

1.4 Therapie

Bei rechtzeitiger Erkennung und konsequenter Allergenkarenz kann die EAA folgenlos abheilen.

- Abwarten des Spontanverlaufs unter strikter Expositions- - Sennekamp H-J: karenz.

- Prednisonbehandlung nur im Schub bei erheblicher Symptomatik und/oder Hypoxämie

1.5 Prävention

Exogen allergische Alveolitis.

Dustri-Verlag, 2004

Allergenkarenz ist unverzichtbar.

- Kontinuierliche Exposition kann zu irreversibler, tödlicher - Sennekamp H-J: Lungenfibrose führen.

- Nur in beruflichen Ausnahmesituationen sind die verbesserten modernen Atemschutzmasken eine Alternative.

Dustri-Verlag, 2004

\section{Lungenfibrosen}

\subsection{Klassifikation}

Statt des früher üblichen „Sammeltopfs - UIP = Usual interstitial pneumonia (eigentlich die alte IPF) der idiopathischen Lungenfibrose“ (IPF) - NSIP = Nonspecific interstitial pneumonia werden heute sieben histologische Entitäten unterschieden. Den morphologischen Befunden werden eigene - COP = Cryptogenic organizing pneumonia (früher als BOOP bezeichnet) Krankheitsbilder mit klinischen, thera-

- DAD = Diffuse alveolar damage peutischen und prognostischen Charakteristika zugeordnet. Warum wir eine neue Klassifikation brauchen, sagt Ihnen Professor Costabel in einem Editorial.

- $\mathrm{RB}=$ Respiratory bronchiolitis (RBILD $=\mathrm{RB}+$ interstitial lung disease)

- DIP = Desquamative interstitial pneumonia

- LIP = Lymphoid interstitial pneumonia

\section{- ATS/ERS :}

International Multidisciplinary Consensus Classification of the Idiopathic Interstitial Pneumonias

Am J Respir Crit Care Med 2002; 165 :

277-304

- Costabel U:

Idiopathische interstitielle Pneumonien wozu schon wieder eine Konsensusklassifikation?

Pneumologie 2002; 56: 279-280 
Die Klassifikation beruht auf einer pathologisch-anatomischen Beschreibung höchst unterschiedlicher Entitäten, die alle letztlich zum uniformen Bild der Lungenfibrose führen. Die Biopsie macht daher nur in frühen Stadien Sinn.

2.3 Diagnostik

Durch die neue Klassifikation der Lungenfibrosen und das graduell sich ausweitende therapeutische Armentarium ist die histologische Sicherung durch die chirurgische Lungenbiopsie wieder in den Vordergrund gerückt. Das HRCT gibt meist wertvolle Hinweise auf die Entität.

\subsection{Therapie}

Zur Beurteilung der Wirksamkeit von Medikamenten ist die korrekte Zuordnung und Typisierung der generalisierten Lungenparenchymerkrankung wegen ihrer unterschiedlichen Verläufe unerlässlich. Die Palette der Medikamente „in der pipeline“ für die frühen Phase ist breit und die Chance hier am größten, den Übergang in das Endstadium der Lungenfibrose zu verhindern.

2.5 Prävention

Nichtrauchen
- Die „Lungenfibrose“ ist die gemeinsame Endstrecke der verschiedenen Erkrankungen, histologisch oft nicht weiter zu differenzieren und therapeutisch kaum beeinflussbar.

- Somit ist eine frühzeitige histologische Sicherung für die rechtzeitige Intervention und damit Prognose wichtig.

- Costabel U, Gutsman J:

Lungenfibrosen, Klassifikation, Diagnostik, Therapie.

Der Internist 2003; 44 (Suppl. 1): S35-43

- Das internationale Konsensusstatement sieht einen diag- - ATS/ERS: nostischen Algorithmus vor, der von der Basisdiagnostik bis zur offenen Lungenbiopsie reicht.

- Zur Basisdiagnostik werden Anamnese, Klinik, Rö-Thorax, Lufu, Laborparameter gerechnet.

- Spezielle Diagnostik: HRCT. Das HRCT hat auch eine prognostische Aussage.

- Die Bronchoskopie mit BAL und transbronchialer Biopsie kann dem CT folgen, das CT bestimmt den Ort der Entnahme.

- Histologisch lassen sich nur 5 der 7 Typen mit Lungenbiopsie sicher klassifizieren.

- Prednison \pm Immunsuppressiva führt zu keiner Besserung, stabilisiert bestenfalls den Verlauf.

$-\gamma$-Interferon führt weder zur funktionellen Besserung noch verlängert es das rezidivfreie Überleben.

- Pirfenidon, ein neues Pharmakon mit antiinflammatorischer, antioxydativer und antifibrotischer Wirkung im Tierversuch vermag bei IPF die Zahl der Schübe zu vermindern und die Lungenfunktion zu verbessern.

- Klinische Studien mit Antioxydantien (N-Acetylcystein) und antifibrotisch wirksamen Substanzen (TNF-alphaAntagonisten, Antiendothelin dual receptor antagonist) stehen kurz vor der Auswertung.

- Die Lungentransplantation ist immer noch eine Option, wenn auch wegen des oft raschen Progresses eine Stabilisierung nicht gelingt. Der Tod auf der Warteliste ist daher häufiger als bei anderen Indikationen.

- Azuma A, Nukiwa T, Tsuboi E et al. Double blind, placebo controlled trial of pirfenidone in patients with idiopathic pulmonary fibrosis.

Am J Resp Crit Care Med 2005; 171 1040 - 1047

- Raghu G, Brown KK, Bradford WZ et al.: A placebo-controlled trial of interferon gamma-1b in patients with idiopathic pulmonary fibrosis.

N Engl J Med 2004; 350: 125-133

- Bewig B:

Idiopathische Lungenfibrose

Der Pneumologe 2005; 2: 457 - 468

\section{- Histiozytosis X, RBILD und DIP werden durch Tabakrauch} induziert oder verschlimmert.

- Schönfeld N:

Pulmonale Langerhans-Zell-Histiozytosis. Pneumologie 2003; 57: 157-163

3. Sarkoidose

\section{1 Ätiologie und Epidemiologie}

Der oder die Auslöser der Sarkoidose sind immer noch ungeklärt, eine kausale Therapie gibt es nicht.
- Die Prävalenz der Sarkoidose liegt bei 50-60/100000, die Sarkoidose ist damit die häufigste interstitielle Lungenerkrankung.

- Die Sarkoidose tritt familiär gehäuft auf.

- Nach neueren molekularbiologischen Erkenntnissen wird generell die Disposition zu granulomatösen Erkrankungen vererbt. 
Aufregend Neues zur Therapie der Sarkoidose gibt es in den letzten Jahren nicht zu berichten, viele anfänglich interessant erscheinenden Behandlungsansätze (Cyclosporin A, Spironolacton) sind wieder verlassen. Neuere prospektive klinische Studien zur Wirksamkeit der Cortisontherapie gibt es nicht, Metaanalysen sprechen jedoch für ihren Effekt in späteren Stadien.
- Topische Steroide haben bei lokalen Komplikationen ihre Indikation: Iridocyclitis. Hautbefall, Husten bei sarkoidbefallener Bronchialschleimhaut.

- Die Indikation zur systemischen Prednisontherapie ist in den letzten Jahren eher geringer geworden, eben so Dauer und Dosierung.

- Zur Prophylaxe der gefürchteten Kortisoninduzierten Osteoporose werden häufig Vitamin D-Präparate eingesetzt werden, was kontraindiziert ist Hyperkalzämie!).
- Deutsche Gesellschaft für Pneumologie: Empfehlungen zur Therapie der Sarkoidose.

Pneumologie 1998; 52: 26-30

$4 \quad$ Seltene Lungenerkrankungen

Lymphangioleiomyomatose (LAM)

Histiozytosis X (Langerhans-Zell-Granulomatose)

Respiratorische Bronchiolitis mit interstitieller Lungenerkrankung (RBILD)

Bronchiolitis obliterans mit organisierender Pneumonie (BOOP; COP)

Eosinophile Pneumonien

Alveolarproteinose

Bronchopulmonale Amyloidose

Bronchozentrische Granulomatose

Lungenhämoside
- Costabel U, Guzman J: Pulmonale Lymphangioleiomyomatose. Pneumologie 2002; 56: 309-315

- Schönfeld N:

Pulmonale Langerhans-Zell-Histiozytosis. Pneumologie 2003; 57: 157 - 163

- Goeckenjan G:

Respiratorische Bronchiolitis mit interstitieller Lungenerkrankung (RB-ILD). Pneumologie 2003; 57: 278-287

- Teschler H, Wessendorf TE: Bronchiolitis obliterans mit organisierender Pneumonie (BOOP).

Pneumologie 2002; 56: 798 - 806

- Kirsten D:

Idiopathische eosinophile Pneumonien. Pneumologie 2002; 56: 621-630

- Teschler H, Wessendorf TE:

Alveolarproteinose. Pneumologie 2002; 56: 448-456

- Konietzko N:

Die Amyloidose der Lunge.

Pneumologie 2004; 58: 339-343

- Westhoff M et al.:

Bronchozentrische Granulomatose -

Seltene Ursache pulmonaler Rundherde. Pneumologie 2004; 58

- siehe Spezialliteratur 